\title{
Analysis of Fifteen Coupled Chaotic Oscillators Composed of RC Circuits
}

\author{
Ayana Shimada $^{\dagger}$, Yoko Uwate ${ }^{\dagger}$, Yoshifumi Nishio ${ }^{\dagger}$ and Jingmin Xin ${ }^{\ddagger}$ \\ $\dagger$ Tokushima University \\ 2-1 Minami-Josanjima, Tokushima 770-8506, Japan \\ Phone: +81-88-656-7470 \\ Email: \{ayana,uwate,nishio\}@ee.tokushima-u.ac.jp \\ $\$ \mathrm{Xi}$ 'an Jiaotong University \\ 28 Xianning Xilu, Xi'an, Shaanxi 710049, China \\ Phone: +86-29-8266-8802 \\ Email: jxin@mail.xjtu.edu.cn
}

\begin{abstract}
In this study, we analyze fifteen coupled chaotic oscillators composed of RC circuits. In particular, we pay our attention on the cross correlation characteristics between neighboring oscillators. In addition, we investigate the cross correlation characteristics between the first oscillator and the others. By computer calculations, we investigate how the cross correlation changes as a circuit parameter increases. Further, the oscillators in the later stage are confirmed to have stronger correlation than those in the early stage.
\end{abstract}

\section{Introduction}

Recently, many researchers have shown their interests in chaotic systems. Chaos has been investigated not only in engineering but also in various fields such as medicine, sociology and economics. In the field of electrical and electronic engineering, researchers have proposed various applications using chaos as chaos communications, chaos cryptosystem, chaos neural networks and so on. In order to realize chaotic engineering systems, it is important to investigate simple chaos-generating circuits.

In our previous study, a simple chaotic oscillator using two RC circuits were proposed [1]. We carried out computer calculations and investigated chaotic behavior when the number of RC circuits increased [2].

In this study, we analyze fifteen coupled chaotic oscillators composed of RC circuits. In particular, we pay our attention on the cross correlation characteristics between neighboring oscillators. In addition, we investigate the cross correlation characteristics between the first oscillator and the others. By computer calculations, we investigate how the cross correlation changes as a circuit parameter increases. Further, the oscillators in the later stage are confirmed to have stronger correlation than those in the early stage.

\section{Circuit model}

Figure 1 shows the circuit model. In this figure 15 chaotic oscillators, which was proposed in [1], are coupled via one-way coupling through simple comparators. An independent rectangular voltage source is connected only to the first oscillator and the voltage across one of the two capacitors in the previous stage of oscillators is inputted to the comparators in the next oscillator. Hence, the whole circuit consists of one rectangular voltage source, 30 comparators, 30 resistors, and 30 capacitors.

Figure 2(a) shows the rectangular voltage waveform $V_{S}(t)$ inputted to the first oscillator. $E \alpha$ is the amplitude of the rectangular voltage and $T$ is the period of the waveform. The circuit equations are described as follows:

$$
\begin{aligned}
& R C \frac{d v_{1}}{d t}= \begin{cases}-v_{1}+E & \left(v_{2}>V_{S}\right) \\
-v_{1}-E & \left(v_{2}<V_{S}\right)\end{cases} \\
& R C \frac{d v_{2}}{d t}= \begin{cases}-v_{2}-E & \left(v_{1}>V_{S}\right) \\
-v_{2}+E & \left(v_{1}<V_{S}\right)\end{cases} \\
& R C \frac{d v_{n}}{d t}= \begin{cases}-v_{n}+E & \left(v_{n+1}>v_{n-1}\right) \\
-v_{n}-E & \left(v_{n+1}<v_{n-1}\right)\end{cases} \\
& R C \frac{d v_{n+1}}{d t}= \begin{cases}-v_{n+1}-E & \left(v_{n}>v_{n-1}\right) \\
-v_{n+1}+E & \left(v_{n}<v_{n-1}\right) \\
(n=3,5,7,9, \cdots, 29)\end{cases}
\end{aligned}
$$

where $E$ is the output voltage of the comparators, namely the DC supply voltage of the operational amplifiers. By using the following variables and the parameters,

$$
\begin{array}{r}
v_{k}=E x_{k}, t=R C \tau, T=R C \beta, \\
(k=1,2,3,4, \cdots, 30)
\end{array}
$$

we obtain the normalized circuit equations. Because the circuit equations are linear in each region, the rigorous solution of the circuit equations can be derived as follows:

$$
\begin{aligned}
& x_{1}= \begin{cases}\left(x_{10}-1\right) e^{-\tau}+1 & \left(x_{2}>V_{\alpha}\right) \\
\left(x_{10}+1\right) e^{-\tau}-1 & \left(x_{2}<V_{\alpha}\right)\end{cases} \\
& x_{2}= \begin{cases}\left(x_{20}+1\right) e^{-\tau}-1 & \left(x_{1}>V_{\alpha}\right) \\
\left(x_{20}-1\right) e^{-\tau}+1 & \left(x_{1}<V_{\alpha}\right)\end{cases} \\
& x_{n}= \begin{cases}\left(x_{n 0}-1\right) e^{-\tau}+1 & \left(x_{n+1}>x_{n-1}\right) \\
\left(x_{n 0}+1\right) e^{-\tau}-1 & \left(x_{n+1}<x_{n-1}\right)\end{cases} \\
& x_{n+1}=\left\{\begin{array}{rr}
\left(x_{(n+1) 0}+1\right) e^{-\tau}-1 & \left(x_{n}>x_{n-1}\right) \\
\left(x_{(n+1) 0}-1\right) e^{-\tau}+1 & \left(x_{n}<x_{n-1}\right) \\
(n=3,5,7,9, \cdots, 29)
\end{array}\right.
\end{aligned}
$$

where $V_{\alpha}$ corresponds to $V_{S}$ and shown in Fig. 2(b), and $x_{10}, x_{20}$ and $x_{n 0}$ are initial values $(n=3,5,7,9, \cdots, 29)$. 

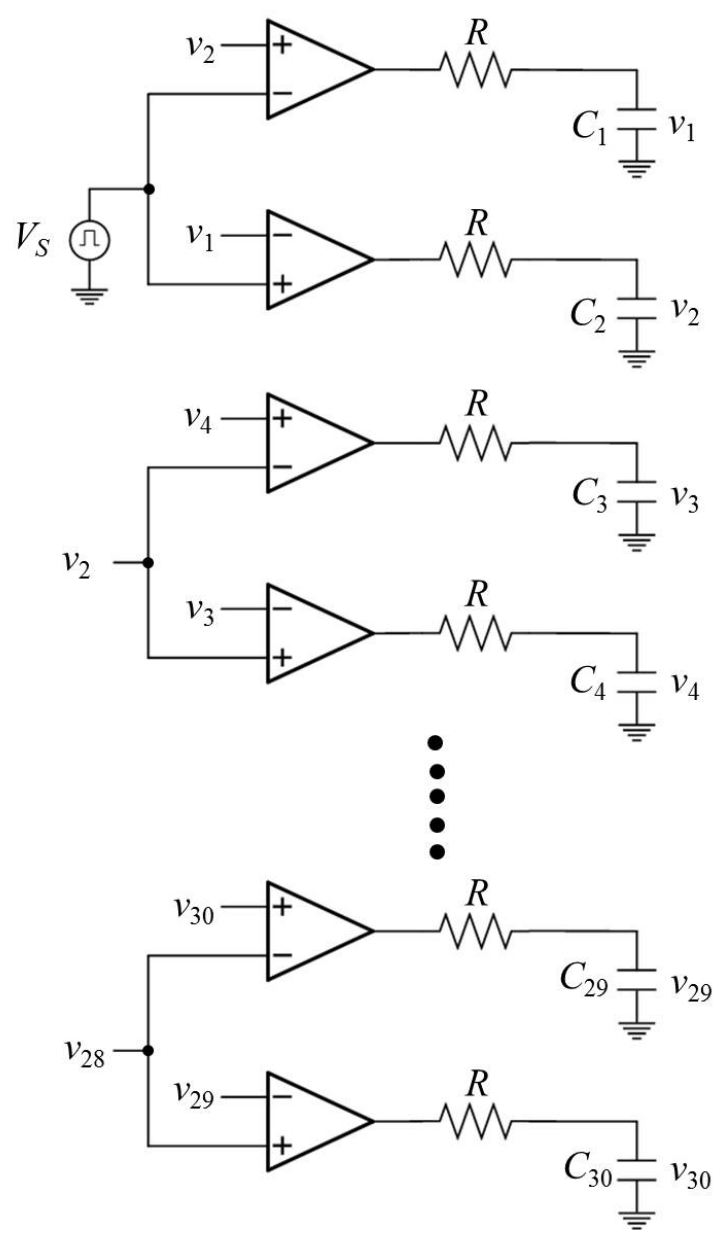

Figure 1: Circuit model.

\section{Simulation results}

Figure 3 shows some examples of chaotic attractors obtained from computer calculations. We compare Fig. 3(a2) with (c2), then we can see that the degree of chaos becomes larger for the later stage. When the value of $\alpha$ becomes larger, only periodic attractors are observed in the early stages as shown in Fig. 3(a3).

Figures 4(a) and (b) show the time waveform, when the values of $\alpha$ are 0.058 and 0.080 , respectively. There exist regular and irregular parts in the waveforms. We pay our attentions on the relation between the time waveforms of neighboring oscillators.

We show the cross correlations of neighboring oscillators in Figs. 5(a) and (b). Vertical axis is the correlation coefficient $R_{x, y}$ between $x_{m}$ and $x_{m+2}(m=1,3,5,7,17,27)$. Horizontal axis shows the delay time $\Delta \tau$. We find that neighboring oscillators correlate from Figs. 5(a) and (b). In Fig. 5(a), when the value of $\Delta \tau$ is 0.18 , the cross correlation coefficient of $x_{1}-x_{3}$ takes the largest value 0.53 . The cross correlation coefficient of $x_{27}-x_{29}$ takes the largest value 0.67 also for $\Delta \tau=0.18$. Therefore, we can say that the cross correlation becomes larger for the later stages. We

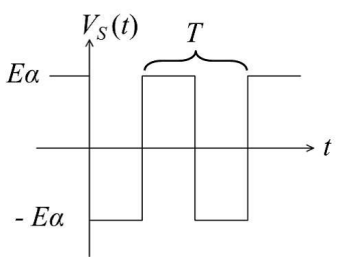

(a)

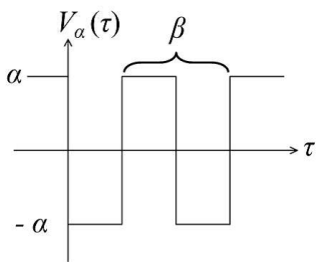

(b)
Figure 2: Rectangular voltage waveform.
(1)
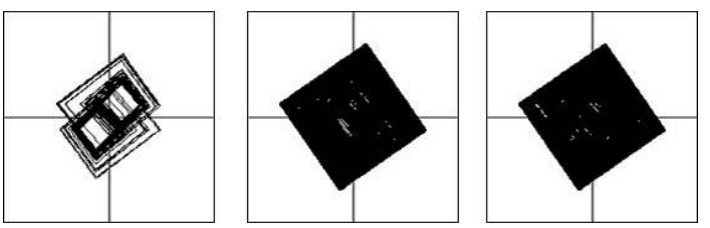

(2)
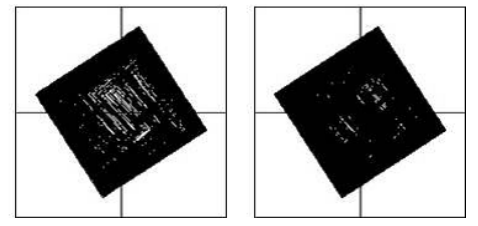

(3)

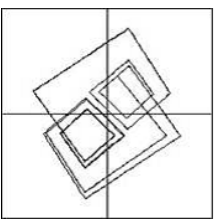

(a)

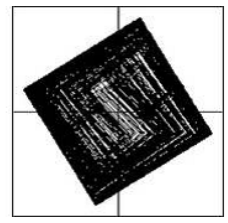

(b)

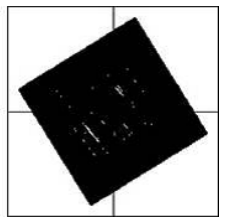

(c)

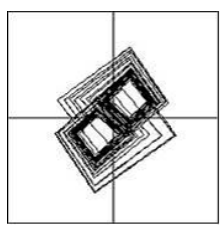

Figure 3: Chaotic attractors obtained from computer calculations for $\beta=4.0$. (a) $x_{1}-x_{3}$, (b) $x_{13}-x_{15}$, and (c) $x_{29}-x_{30}$. (1) $\alpha=0.058$, (2) $\alpha=0.080$, and (3) $\alpha=0.100$.

summarize the values of $\Delta \tau$ for which the cross correlation coefficient takes the maximum value $\max \left(R_{x y}\right)$ in Table 1. We find that the neighboring oscillators have strong positive correlations.

Figures 6 and 7 show the attractors reconstructed by considering the time delay $\Delta \tau$ shown in Table 1 . From the shapes of these attractors, we can also see that the neighboring oscillators are correlated with some amount of time delay.

Next, Fig. 8 shows the cross correlations of the first oscillator and others. Vertical axis is the correlation coefficient $R_{x, y}$ between $x_{1}$ and $x_{l}(l=3,5,11,17,23,29)$. Horizontal axis shows the delay time $\Delta \tau$. We can see that the correlation coefficients between $x_{1}$ and others are very small except $x_{3}$ and $x_{5}$. This means that the first oscillator does not have strong correlations with the oscillators on the later stages.

Figure 9 shows the attractors reconstructed by considering the appropriate time delay obtained from Fig. 8. We cannot see any strong correlations. 


(n)

(a)

(n)

(b)

Figure 4: Time waveforms for $\beta=4.0$. From top: $x_{1}$, $x_{3}, x_{5}, x_{7}, x_{9}, x_{11}, x_{13}, x_{15}, x_{17}, x_{19}, x_{21}, x_{23}, x_{25}, x_{27}, x_{29}$. (a) $\alpha=0.058$ and (b) $\alpha=0.080$.

Table 1: Time delay $\Delta \tau$ with the maximum values of correlation coefficient $\max \left(R_{x y}\right)$.

\begin{tabular}{|c|c|c|c|c|}
\hline \multirow{2}{*}{} & \multicolumn{2}{|c|}{$\alpha=0.058$} & \multicolumn{2}{c|}{$\alpha=0.080$} \\
\cline { 2 - 5 } & $\Delta \tau$ & $\max \left(R_{x, y}\right)$ & $\Delta \tau$ & $\max \left(R_{x, y}\right)$ \\
\hline \hline$x_{1}-x_{3}$ & 0.18 & 0.53 & 0.20 & 0.63 \\
$x_{3}-x_{5}$ & 0.22 & 0.64 & 0.20 & 0.56 \\
$x_{5}-x_{7}$ & 0.21 & 0.61 & 0.22 & 0.61 \\
$x_{7}-x_{9}$ & 0.18 & 0.54 & 0.26 & 0.72 \\
$x_{17}-x_{19}$ & 0.21 & 0.70 & 0.20 & 0.66 \\
$x_{27}-x_{29}$ & 0.18 & 0.67 & 0.21 & 0.73 \\
\hline
\end{tabular}

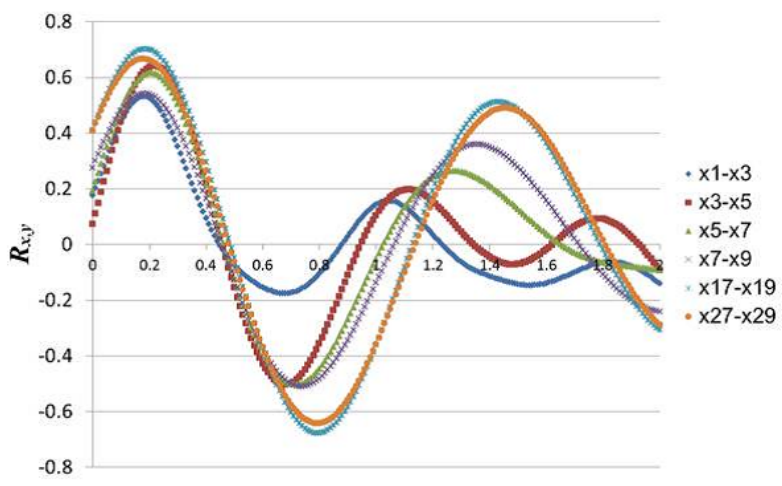

(a)

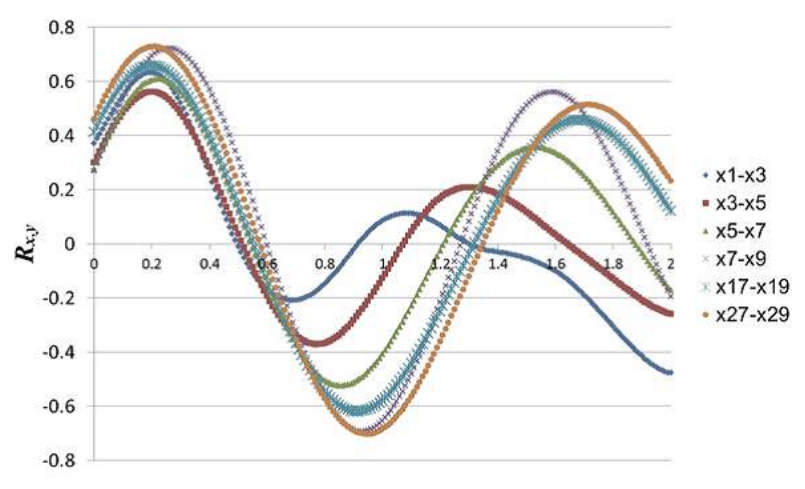

(b)

Figure 5: Graph of the cross correlation value of neighboring oscillators for $\beta=4.0$. (a) $\alpha=0.058$ and (b) $\alpha=0.080$.

\section{Conclusion}

In this study, we have analyzed fifteen coupled chaotic oscillators composed of RC circuits. In particular, we paid our attention on the cross correlation characteristics between neighboring oscillators. In addition, we investigated the cross correlation characteristic between the first oscillator and others. By computer calculations, we investigated the cross correlation changes as a circuit parameter increases. We could observe the cross correlation characteristic between neighboring oscillators. However, we found that non-adjacent oscillator does not have correlation. The chaotic oscillators in the later stage were confirmed to have stronger correlation than those in the early stage.

In our future work, we will subject detailed research of the cross correlation of neighboring oscillators and analyze with Lyapunov exponent.

\section{Acknowledgments}

This work was partly supported by JSPS Grant-in-Aid for for Scientific Research 22500203. 


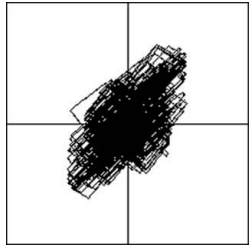

(a)

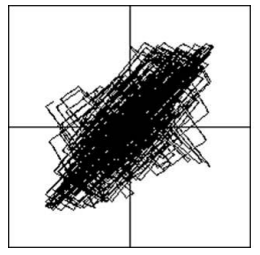

(d)

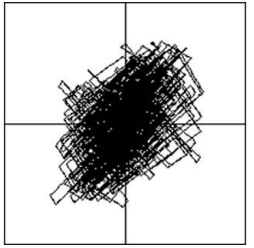

(b)

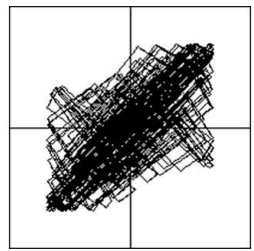

(e)

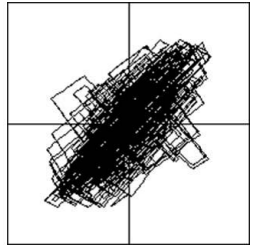

(c)

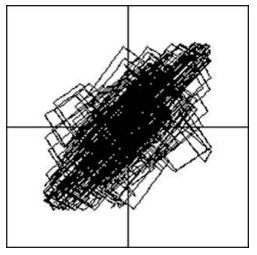

(f)
Figure 6: Attractors reconstructed with time delay for $\alpha=$ 0.058 and $\beta=4.0$. (a) $x_{1}(\tau)-x_{3}(\tau+0.18)$, (b) $x_{3}(\tau)-$ $x_{5}(\tau+0.22)$, (c) $x_{5}(\tau)-x_{7}(\tau+0.21),(\mathrm{d}) x_{7}(\tau)-x_{9}(\tau+0.18)$, (e) $x_{17}(\tau)-x_{19}(\tau+0.21)$, and (f) $x_{27}(\tau)-x_{29}(\tau+0.18)$.

\section{References}

[1] S. Masuda, Y. Uchitani and Y. Nishio, "Simple Chaotic Oscillator Using Two RC Circuits," Proc. of NCSP'09, pp. 89-92, 2009.

[2] A. Shimada, H. Kumeno, Y. Uwate, Y. Nishio and J. Xin, "Coupled Chaotic Oscillators Composed of RC Circuits," Proc. of NCSP'12, pp. 17-20, 2012.

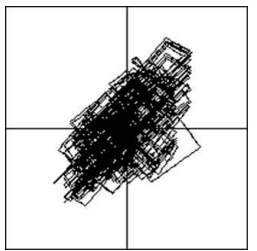

(a)

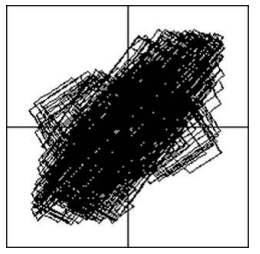

(d)

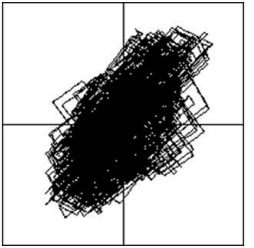

(b)

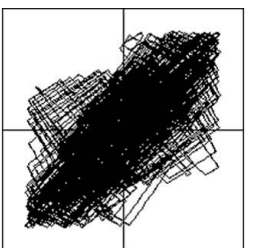

(e)

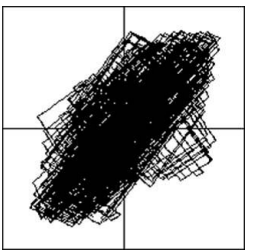

(c)

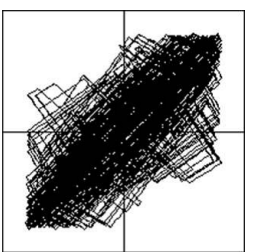

(f)
Figure 7: Attractors reconstructed with time delay for $\alpha=$ 0.080 and $\beta=4.0$. (a) $x_{1}(\tau)-x_{3}(\tau+0.20)$, (b) $x_{3}(\tau)-$ $x_{5}(\tau+0.20)$, (c) $x_{5}(\tau)-x_{7}(\tau+0.22)$, (d) $x_{7}(\tau)-x_{9}(\tau+0.26)$, (e) $x_{17}(\tau)-x_{19}(\tau+0.20)$, and (f) $x_{27}(\tau)-x_{29}(\tau+0.21)$.

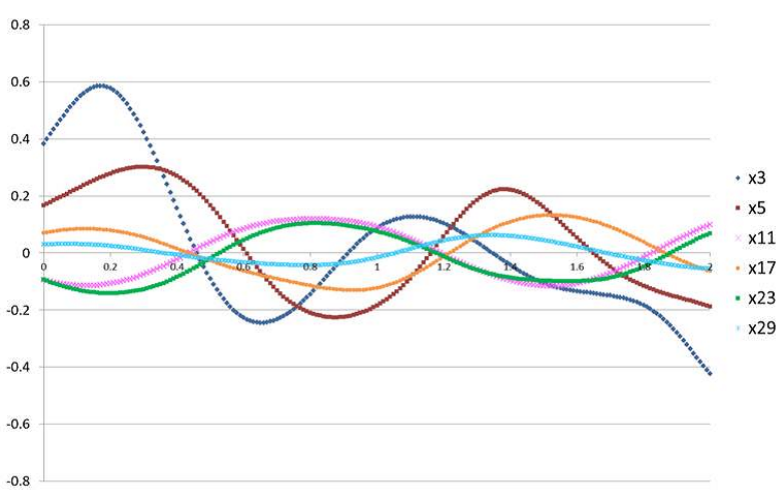

Figure 8: Cross correlation values of the first oscillator and others for $\alpha=0.080$ and $\beta=4.0$.

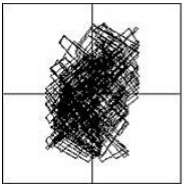

(a)

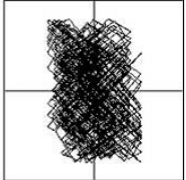

(b)

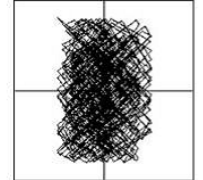

(c)

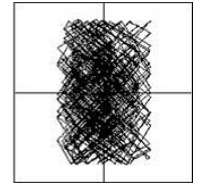

(d)
Figure 9: Attractors reconstructed with time delay for $\alpha=$ 0.080 and $\beta=4.0$. (a) $x_{1}(\tau)-x_{5}(\tau+0.30)$, (b) $x_{1}(\tau)-x_{9}(\tau+$ 1.33), (c) $x_{1}(\tau)-x_{17}(\tau+0.17)$, and (d) $x_{1}(\tau)-x_{29}(\tau+1.35)$. 